\title{
Comparison Study between Extracorporeal and Intracorporeal Anastomosis for Laparoscopic Right Hemicolectomy
}

\author{
Badawy M. Ahmed ${ }^{*}$, Ebrahim Aboeleuon'1, Ahmed Soliman² \\ ${ }^{1}$ Surgical Oncology Department, South Egypt Cancer Institute, Assiut University, Assiut, Egypt \\ ${ }^{2}$ General Surgery Department, Assiut University Hospital, Assiut University, Assiut, Egypt \\ Email: *badawy_znatyy@yahoo.co.uk
}

How to cite this paper: Ahmed, B.M., Aboeleuon, E. and Soliman, A. (2019) Comparison Study between Extracorporeal and Intracorporeal Anastomosis for Laparoscopic Right Hemicolectomy. Journal of Cancer Therapy, 10, 796-805.

https://doi.org/10.4236/jct.2019.1010067

Received: August 27, 2019

Accepted: October 5, 2019

Published: October 8, 2019

Copyright () 2019 by author(s) and Scientific Research Publishing Inc. This work is licensed under the Creative Commons Attribution International License (CC BY 4.0).

http://creativecommons.org/licenses/by/4.0/

\begin{abstract}
Background: Nowadays, laparoscopic colectomy is considered a safe and effective surgical technique regarding short- and long-term outcomes, as well as specific oncologic outcomes. The anastomosis can be created intra- or extracorporeally. The goal of our study was to evaluate and compare short term outcomes of extra- and intra-corporeal anastomosis after laparoscopic right hemi colectomies. Aim of the Study: The goal of our study was to evaluate and compare short term outcomes of extra- and intra-corporeal anastomosis after laparoscopic right hemi colectomies. Methods: In the period from December 2014 to January 2019, all patients underwent laparoscopic right hemicolectomy for cancer colon who presented to surgical oncology department-south Egypt cancer institute and general surgery department-Assiut University was analyzed. Data like age, sex, body mass index (BMI), operative technique, operative times, blood loss, intra- and post-operative complications, pathology and hospital stay were reported and analyzed. Results: Twenty three (69.7\%) patients underwent extracorporeal anastomosis while intracorporeal anastomosis was performed in ten (30.3\%) patients. There was no significant difference in patient characteristics and demographic data in both groups $(P>0.05)$. There was no statistically significant difference in operative (operative time, blood loss or length of hospital stay) and postoperative (ileus, anastomotic leak, wound infection, incisional hernia, readmission, reoperation or deaths) details in the 2 groups except in length of the incision which was significantly shorter in the IA group $(5.500 \pm 1.269)$ vs. $(6.565 \pm 1.308)$ for EA $(P=0.015)$. Conclusion: No significant difference in short term outcomes of laparoscopic-assisted and total laparoscopic right colectomy. Intracorporeal anastomosis had shorter incision which may decrease wound-related complications.
\end{abstract}




\section{Keywords}

Extracorporeal Anastomosis, Intracorporeal Anastomosis, Laparoscopic Right Hemicolectomy

\section{Background}

When discussing colon resection laparoscopic resection is more superior to open surgery regarding postoperative pain, hospital stay and recovery [1]-[4]. However, there are lacking data on technique-specific outcomes and also there are no standardized techniques.

There are many terms used for laparoscopic colon surgery, for example, laparoscopic colectomy with intracorporeal anastomosis (LCIA), hand-assisted colectomy (HAC or HALS), and laparoscopic-assisted colectomy (LAC, usually with extracorporeal anastomosis) [4]-[8]. Also, there are different techniques for mobilization of the mesentery (lateral-to-medial versus medial-to-lateral) and ligation of the vessels (extracorporeal vs. intracorporeally). In most centers, laparoscopic-assisted colectomy (LAC) with extracorporeal ileocolonic anastomosis (EA) for right or extended right colectomies remains the preferred approach [4] [5] [9] [10] [11] [12] [13]. But, this technique has limitation for extraction site, which is usually midline incision. In addition, after extraction sometimes there are problems with intestinal alignment. A total intracorporeal anastomosis (IA) may reduce intestinal twists and give the ability to choose the site for specimen extraction. When comparing Intra-corporeal (IA) with extracorporeal (EA) techniques; intra-corporeal (IA) anastomosis requiring advanced training in order to achieve expertise in laparoscopic manual sutures, and a longer learning curve [14]. With growing enthusiasm about minimally invasive approaches attracts surgeons to develop totally intra-corporeal anastomotic techniques, its theoretical advantages are the easier handling of structures, the ability to choose the incision site for specimen extraction and lower risk of mesenteric torsion. However, according to some authors, there are some disadvantages, such as higher risk of fecal contamination, longer operative time and a more demanding technique [15].

\section{Patients and Methods}

\subsection{Patients}

In the period from December 2014 to January 2019, all patients underwent laparoscopic right hemicolectomy for cancer colon who presented to surgical oncology department-South Egypt Cancer Institute and general surgery department-Assiut university hospital-Assiut university were analyzed. Data like age, sex, body mass index (BMI), operative technique, operative times, blood loss, intra- and post-operative complications, pathology and hospital stay were reported and analyzed. During the study period, 33 cases of laparoscopic right 
hemi colectomies (extracorporeal (EA, $\mathrm{n}=23$ ) anastomosis \& intracorporeal (IA, $\mathrm{n}=10)$ anastomosis). Inclusion criteria were as follows: clinically fit patients with histologically confirmed, clinically stage- 1 to stage- 2 adenocarcinoma of right colon and caecum; no evidence of extra-clonic extension or distant metastasis by means of CT, abdominal ultrasound and chest radiograph. Exclusion criteria were distant metastasis or pre and intra-operative evidence of peritoneal dissemination. All patients in our study underwent elective surgery (no emergency cases) and all patients have colonic mechanical chemical preparation. Also, all have postopertaive prophylactic antibiotic and anticoagulant. Cases that were converted from a laparoscopic to an open approach were not included, as conversion rate was not an item of comparison in our study. Any intention to treat analysis could not be performed because of the retrospective nature of the study. The choice of anastomosis was the surgeon's preference. All patients had preoperative colonoscopies and biopsy. Postoperative ileus was defined as abdominal distension requiring radiological imaging, stoppage of oral feeding and conversion to an NPO-status and placement of a nasogastric tube for decompression.

\subsection{Techniques}

According to the surgeon's preference, pneumoperitoneum was done either via direct insertion of safety trocar or Veress needle. Four to five ports were used: a $10-\mathrm{mm}$ to $12-\mathrm{mm}$ umbilical port for a 0 -degree laparoscope camera, in the left lower abdomen, 10-mm port for stapling devices was used. In the left upper abdomen and suprapubic region another 2 to 3 five-mm working ports located. Mesenteric mobilization was carried out from medial-to-lateral fashion in almost all cases using sealing device.

In the EA group, both right branch of the middle colic artery and ileocolic pedicle were divided close to their origin intra-corporeally with hemoclips in 13 patients. While in the remaining 10 cases, branches of the middle colic artery and the remaining mesentery were ligated after exteriorization the colon whereas, the ileocolic pedicle was divided intra-corporeally. After sufficient mobilization of the right colon and extension of the umbilical port from $4-\mathrm{cm}$ to $8 \mathrm{~cm}$ longitudinally in midline; exteriorization of the colon and resection was done. Externally, a side-to-side, stapled ileocolonic anastomosis was carried out in 7 cases with a double-layer, hand-sewn closure of the enterotomy and an end to end double-layer, hand-sewn ileocolonic anastomosis was created in the remaining 16 cases. The anastomosis was evaluated after closure of the wound and after reinsertion of laparoscope also to exclude twisting of the intestine and assure haemostasis.

In the IA group, after intra-corporial complete mobilization of the colon from medial to lateral and control of ileocolic pedicle and middle colic branches using hemoclips and sealing device, the transverse colon and the terminal ileum were divided intra-corporeally with a $60-\mathrm{mm}$ Endo-GIA stapler. After resection of the 
tumor, the specimen was put in the pelvis and an intra-corporeal ileocolonic side-to-side, isoperistaltic anastomosis was created using 60-mm Endo-GIA. The enterotomy was then closed laparoscopically with a 2-layer, running suture with 3.0 Vicryl. After that, the specimen was retrieved through Pfannenstiel incision and always opened on the side table to ensure that the tumor was included in the resection. Bags for specimen extraction were not used in our study. Patients were followed for 6 months postoperatively.

\subsection{Statistical Analysis}

IBM SPSS Statistics $v 22^{\circledR}$ software was used for statistical analysis. The categorical variables are expressed as mean \pm standard deviation and continuous variables as $\mathrm{n}$ and percentage (\%). Statistically significant differences were assessed with Chi-square test. $\mathrm{P} \leq 0.05$ was considered significant.

\section{Results}

In the period from December 2014 to January 2019, 33 patients (20 males \& 13 females) underwent laparoscopic right hemicolectomy for colonic adenocarcinoma in surgical oncology department-south Egypt cancer institute and general surgery department-Assiut university hospital-Assiut university.

Twenty three (69.7\%) patients underwent extracorporeal anastomosis while intra-corporeal anastomosis was performed in ten (30.3\%) patients. In Table 1, sex distribution for EA group was ( 15 males \& 8 females) and ( 5 males \& 5 females) for IA group, mean age for EA group was $53.869 \pm 10.562$ and $48.800 \pm$ 12.506 for IA group. When comparing BMI for EA and IA groups it was 29.524 \pm 5.830 and $28.480 \pm 6.110$ respectively. Median ASA score was $3(2-4)$ and 3

Table 1. Patient characteristic and demographic data.

\begin{tabular}{cccc}
\hline & EA $^{*} \mathbf{n}=\mathbf{2 3}$ & IA $^{*} \mathbf{n}=10$ & P-value Chi-square \\
\hline Sex & & & \\
Male & 15 & 5 & 0.411 \\
Female & 8 & 5 & \\
Age (years) & & & 0.794 \\
Mean & $53.869 \pm 10.562$ & $48.800 \pm 12.506$ & \\
Range & $25-66$ & $24-62$ & 0.775 \\
BMI & & & \\
Mean & $29.524 \pm 5.830$ & $28.480 \pm 6.110$ & NS \\
Range & $22.7-43$ & $22.9-43$ & NS \\
Diabetes mellitus & $5(21.74 \%)$ & $3(30 \%)$ & NS \\
Hypertension & $7(30.43 \%)$ & $3(30 \%)$ & 0.840 \\
Previous abdominal surgery & $4(17.4 \%)$ & $2(20 \%)$ & $3(2-4)$ \\
ASA score + & $3(2-4)$ & & \\
\hline
\end{tabular}

${ }^{\star}$ EA: Extracorporeal Anastomosis; IA: Intracorporeal Anastomosis; ASA: American Society of Anesthesiology Score. ${ }^{+}$Median values. 
(2 - 4) for EA and IA groups respectively. There was no significant difference in patient characteristics and demographic data between both groups $(\mathrm{P}>0.05)$. There was no statistically significant difference in operative (operative time, blood loss or length of hospital stay) and postoperative (ileus, anastomotic leak, wound infection, incisional hernia, readmission, reoperation or deaths) details between the 2 groups except in length of the incision which was significantly shorter in the IA group $(5.500 \pm 1.269)$ vs. $(6.565 \pm 1.308)$ for EA $(P=0.015)$ (Table 2 and Table 3). Even in pathological details (tumor stage, tumor grade or number of harvested nodes) there was no statistical difference between the 2 groups $(\mathrm{P}>0.05)$ (Table 4$)$.

All patients $9(27.27 \%)$ with ileus were successfully treated conservatively by stopping oral feeding, IV fluids and insertion of nasogastric tube. In the EA group, anastomotic leak was experienced in 3 (13\%) patients, one of those patients had high output fistula and were re-explored and treated by repair of the defect and an end-ileostomy. The other two had low output fistula and treated conservatively by stopping of oral feeding, parenteral nutrition, covering antibiotics and

Table 2. Operative data.

\begin{tabular}{cccc}
\hline & EA $^{*} \mathbf{n}=\mathbf{2 3}$ & IA $^{*} \mathbf{n}=10$ & P-value Chi-square \\
\hline Operative time (min) & & & \\
Mean & $135.869 \pm 18.626$ & $136.000 \pm 15.951$ & 0.970 \\
Range & $110-170$ & $110-160$ & \\
Incision length (cm) & & & $0.015^{*}$ \\
Mean & $6.565 \pm 1.308$ & $5.500 \pm 1.269$ & \\
Range & $4-6$ & $4-8$ & 0.365 \\
EBL (mL) & & & \\
Mean & $66.087 \pm 19.940$ & $52.000 \pm 11.105$ & \\
Range & $30-100$ & $30-70$ & \\
Hospital stay (days) & & & \\
Mean & $12.173 \pm 3.961$ & $10.700 \pm 1.888$ & \\
Range & $8-25$ & $8-15$ & \\
\hline
\end{tabular}

$\mathrm{EA}=$ extracorporeal anastomosism; IA = intracorporeal anastomosis. ${ }^{\star}$ Significant correlation.

Table 3. Postoperative data.

\begin{tabular}{cccc}
\hline & EA $^{*} \mathbf{n}=\mathbf{2 3}$ & IA $^{*} \mathbf{n}=\mathbf{1 0}$ & P-value $^{(0.697) *}$ \\
\hline Ileus & $6(26.1 \%)$ & $3(30 \%)$ & NS \\
Anastomotic leak & $3(13 \%)$ & $0(0.00 \%)$ & NS \\
Leakage \& wound infection & $3(13 \%)$ & $0(0.00 \%)$ & NS \\
Wound infection & $2(8.7 \%)$ & $1(10 \%)$ & NS \\
Incisional hernia & $3(13 \%)$ & $1(10 \%)$ & NS \\
Readmission & $2(8.7 \%)$ & $0(0.00 \%)$ & NS \\
Reoperation & $2(8.7 \%)$ & $0(0.00 \%)$ & NS \\
Mortality & $1(4.35 \%)$ & $1(10 \%)$ & NS
\end{tabular}

${ }^{\star} \mathrm{EA}=$ extracorporeal anastomosis; IA = intracorporeal anastomosis; NS = not significant. 
Table 4. Pathology.

\begin{tabular}{cccc}
\hline & EA $^{*} \mathbf{n}=\mathbf{2 3}$ & IA $^{*} \mathbf{n}=\mathbf{1 0}$ & P-Value \\
\hline Stage & & & \\
I & $4(17.4 \%)$ & $4(40 \%)$ & 0.329 \\
II & $7(30.43 \%)$ & $3(30 \%)$ & \\
III & $12(52.17 \%)$ & $3(30 \%)$ & \\
Harvested LNs & & & 0.722 \\
Mean & $6.521 \pm 2.591$ & $8.800 \pm 3.765$ & \\
Range & $3-12$ & $3-15$ & \\
Grade & & & 0.299 \\
Well. diff & $7(30.43 \%)$ & $1(10 \%)$ & \\
Mod. diff & $10(43.47 \%)$ & $4(40 \%)$ & \\
Poor. diff & $6(26.1 \%)$ & $5(50 \%)$ & \\
\hline
\end{tabular}

drainage of the leak. Fistula successfully healed in one patient within 10 days while in the other one fistula transformed to high output fistula; so the patient underwent reexploration and end-ileostomy but the patient died later on from toxemia and multi-organ failure (the patient was old, $\uparrow \uparrow \uparrow ~ B M I$ and diabetic). In the IA group, there was no leakage. Anastomotic leak was diagnosed before removal of the abdominal drains. Ileostomy was closed after completion of adjuvant chemical therapy. In case of wound infection, there were 5 patients in EA group, three of them have anastomotic leak and only one case in IA group. Incisional hernia was present in four (12.12\%) patients; 3 in EA and 1 in IA group all of them have previous wound infection and high BMI; repair was done 6 months after surgery after completion of medical oncology treatment. Readmission after discharge happened in $2(6 \%)$ cases only in EA group because of burst abdomen.

Two deaths (overall 30-day mortality 6\%) occurred in our series one in the EA group (were described before) and one in IA group due to DVT and pulmonary embolism in spite of anticoagulant (the patient was old, cardiac and $\uparrow \uparrow \uparrow \mathrm{BMI}$ ).

\section{Discussion}

Several studies and trials prove the feasibility, safety and efficacy of laparoscopic colectomy [14] [15] [16] [17].

From the advantages of the laparoscopic approach; reduced intra-operative blood loss, reduced postoperative pain, and decreased rate of ileus, enhanced recovery and lower overall morbidity. From all the above the concept that total laparoscopic colectomy with intra-corporeal anastomosis may have more advantages. Four series present their experience with ileocolonic intra-corporeal anastomosis [7] [8] [18] [19]. The largest series presented by Franklin et al. [8] comparing 10 cases of extra-corporeal anastomosis with 82 cases of intra-corporeal anastomosis for right colon resections. They found that intra-corporeal approach was feasible and safe with no significant difference in operative times and complication rates. This was confirmed by Bergamaschi et al. [19] who evaluate 111 cases of intra-corporeal right colectomies for short-term outcomes. All pa- 
tients in our study had adenocarcinoma and underwent elective surgery. After analysis of the data in our study, there was no statistical difference between the two groups regarding demographic data. Also, there was no significant difference in operative details; mean operative time and mean EBL was $135.869 \pm$ 18.626 and $66.087 \pm 19.940$ for EA group respectively versus $136.000 \pm 15.951$ and $52.000 \pm 11.105$ for IA group respectively. This agrees with Hanna et al. [20] who studied patients from 2005 to 2014 and found an improvement in median operative time from 240 minutes to 170 minutes, without statistically significant difference in the IA group. And with Ines Campos Gil et al. [21] who studied 115 patients.

But there is significant difference in mean length of the incision between EA $(6.565 \pm 1.308 \mathrm{~cm})$ and $\mathrm{IA}(5.500 \pm 1.269 \mathrm{~cm}) \mathrm{P}=0.015$.

In obese patients; small bowel mesentery may be short and thick so that the terminal ileum could not be exteriorized adequately. Thus intracorporeal anastomosis in these patients is more easily.

In our study there were no significant statistical differences between EA and IA in pathological parameters (tumor stage, grade and number of harvested lymph nodes) $\mathrm{P}$-value $>0.05$.

About postoperative outcomes and complications, there were no significant statistical differences between the two groups P-value $=0.697$.

Incidence of complications in our study as follow; ileus 9 (27.27\%) cases (6 cases in EA \& 3 cases in IA group), leakage 3 (9.1\%) cases (the 3 cases in EA group with no cases in IA group this make tendency toward lower incidence of anastomotic in failure IA in spite not reaching statistically significant difference which may be explained by good delineation of tissue under direct vision, low risk of mesenteric torsion and low tension at anastomotic line). A retrospective study done by Fabozzi et al. [22] including 50 patients found significant decrease in anastomotic leak $(\mathrm{p}<0.05)$. However, retrospective study published by Hannah et al. [20], which included 109 patients in the EA group and 86 patients in the IA, reporting a lower anastomotic leak in the IA group that did not reach statistical significance $(\mathrm{p}>0.05)$. A meta-analysis [23] of 484 patients (including 272 patients in the IA group and 212 patients in the EA group) found no statistically significant difference in anastomotic leak between the two groups. From this, the effect of the IA technique on anastomosis remains unclear, wound infection 6 (18.2\%) patients (5 patients in EA \& one patient in IA group). However, Ines Campos Gil et al. [21] report significant statistical difference in wound infection between EA group $21.4 \%$ and IA group 3.2\% $(\mathrm{P}<0.05)$, readmission and reoperation 2 cases in EA group only and incisional hernia happened in $4(12.12 \%)$ patients $3(9.1 \%)$ in EA and 1(3\%) in IA group. In other studies [24] [25], incisional hernia rate was as high as $17 \%$ to $24 \%$ with a higher rate for midline but in another study using the Pfannenstiel incision; incisional hernia rate was as low as $0 \%$ to $2 \%$ [26] [27] [28]. In spite of the increased range of hospital stay in EA group but there was no significant statistical difference in 
the mean of hospital stay $12.173 \pm 3.961$ vs. $10.700 \pm 1.888$ for EA and IA group respectively $(\mathrm{P}=0.949)$. Perioperative mortality rate in our study happened in one patient of EA group representing $4.35 \%$ of this group and was due to anastomotic leakage and multi-organ failure also in IA death happened in $1(10 \%)$ patient and was due to DVT and pulmonary embolization; the difference was not statistically significant.

\section{Conclusion}

Our study and most other similar studies found no significant difference in short term outcomes of laparoscopic-assisted and total laparoscopic right colectomy. However, there was tendency toward smaller incision in the IA group (though affect patient perception of pain) with tendency toward more anastomosis-related complications in the EA group but without statistical significance. In obese patients, small bowel mesentery may be short and thick so that the terminal ileum could not be exteriorized adequately. Thus intra-corporeal anastomosis in these patients is more easily. Because of small number of cases in our study, larger prospective trials are necessary to confirm these findings.

\section{Limitation}

Small number of the study especially total laparoscopic group as we have limited financial supplementation and this is a coasty operation in developing country.

\section{Ethical Considerations}

Approval for this study was obtained from our Ethical Committee.

\section{Conflicts of Interest}

The authors declare no conflicts of interest regarding the publication of this paper.

\section{References}

[1] Reza, M.M., et al. (2006) Systematic Review of Laparoscopic versus Open Surgery for Colorectal Cancer. British Journal of Surgery, 93, 921-928. https://doi.org/10.1002/bjs.5430

[2] Milsom, J.W., et al. (2001) Prospective, Randomized Trial Comparing Laparoscopic vs. Conventional Surgery for Refractory Ileocolic Crohn's Disease. Diseases of the Colon \& Rectum, 44, 1-8. https://doi.org/10.1007/BF02234810

[3] Solomon, M.J., et al. (2002) Randomized Clinical Trial of Laparoscopic versus Open Abdominal Rectopexy for Rectal Prolapse. British Journal of Surgery, 89, 35-39. https://doi.org/10.1046/j.0007-1323.2001.01957.x

[4] The Clinical Outcomes of Surgical Therapy Study Group (2004) A Comparison of Laparoscopically Assisted and Open Colectomy for Colon Cancer. The New England Journal of Medicine, 350, 2050-2059. https://doi.org/10.1056/NEJMoa032651

[5] Baca, I., Perko, Z. and Bokan, I. (2005) Technique and Survival after Laparoscopically Assisted Right Hemicolectomy. Surgical Endoscopy, 19, 650-655. 
https://doi.org/10.1007/s00464-004-9068-6

[6] Ballantyne, G.H. and Leahy, P.F. (2004) Hand-Assisted Laparoscopic Colectomy: Evolution to a Clinically Useful Technique. Diseases of the Colon \& Rectum, 47, 753-765. https://doi.org/10.1007/s10350-003-0108-4

[7] Casciola, L., Ceccarelli, G. and Di Zitti, L. (2003) Laparoscopic Right Hemicolectomy with Intracorporeal Anastomosis. Technical Aspects and Personal Experience. Minerva Chirurgica, 58, 621-627. (In Italian)

[8] Franklin Jr., M.E., Gonzalez Jr., J.J. and Miter, D.B. (2004) Laparoscopic Right Hemicolectomy for Cancer: 11-Year Experience. Revista de Gastroenterología de México, 69, 65-72.

[9] Kaiser, A.M., et al. (2004) Laparoscopic-Assisted vs. Open Colectomy for Colon Cancer: A Prospective Randomized Trial. Journal of Laparoendoscopic \& Advanced Surgical Techniques A, 14, 329-334. https://doi.org/10.1089/1092642042728139

[10] Leung, K.L., et al. (1999) Laparoscopic-Assisted Resection of Right-Sided Colonic Carcinoma: A Case-Control Study. Journal of Surgical Oncology, 71, 97-100. https://doi.org/10.1002/(SICI)1096-9098(199906)71:2<97::AID-JSO7>3.3.CO;2-E

[11] Lezoche, E., et al. (2002) Laparoscopic vs. Open Hemicolectomy for Colon Cancer. Surgical Endoscopy, 16, 596-602. https://doi.org/10.1007/s00464-001-9053-2

[12] Senagore, A.J., et al. (2004) Standardized Approach to Laparoscopic Right Colectomy: Outcomes in 70 Consecutive Cases. Journal of the American College of Surgeon, 199, 675-679. https://doi.org/10.1016/j.jamcollsurg.2004.06.021

[13] Senagore, A.J. and Delaney, C.P. (2006) A Critical Analysis of Laparoscopic Colectomy at a Single Institution: Lessons Learned after 1000 Cases. The American Journal of Surgery, 191, 377-380. https://doi.org/10.1016/j.amjsurg.2005.10.039

[14] Jamali, F.R., et al. (2008) Evaluating the Degree of Difficulty of Laparo-Scopic Colorectal Surgery. The Archives of Surgery, 143, 762-767.

https://doi.org/10.1001/archsurg.143.8.762

[15] Carmichael, J.C. and Stamos, M.J. (2015) Right Hemicolectomy and Ileocecectomy: Laparoscopic Approach. In: Bardakcioglu, Ed., Advanced Techniques in Minimally Invasive and Robotic Colorectal Surgery, Springer, New York, 37-48. https://doi.org/10.1007/978-1-4899-7531-7_5

[16] Kuhry, E., et al. (2008) Long-Term Results of Laparoscopic Colorectal Cancer Resection. Cochrane Database of Systematic Reviews, No. 2, CD003432. https://doi.org/10.1002/14651858.CD003432.pub2

[17] Clinical Outcomes of Surgical Therapy Study Group (2004) A Comparison of Laparoscopically Assisted and Open Colectomy for Colon Cancer. Annals of Surgery, 240, 205-213.

[18] Guillou, P.J., et al. (2005) Short-Term Endpoints of Conventional versus Laparoscopic-Assisted Surgery in Patients with Colorectal Cancer (MRC CLASICC Trial): Multicentre, Randomised Controlled Trial. The Lancet, 365, 1718-1726. https://doi.org/10.1016/S0140-6736(05)66545-2

[19] Colon Cancer Laparoscopic or Open Resection Study Group, Buunen, M., et al. (2009) Survival after Laparoscopic Surgery versus Open Surgery for Colon Cancer: Long-Term Outcome of a Randomised Clinical Trial. The Lancet Oncology, 10, 44-52. https://doi.org/10.1016/S1470-2045(08)70310-3

[20] Raftopoulos, I., Courcoulas, A.P. and Blumberg, D. (2006) Should Completely Intracorporeal Anastomosis Be Considered in Obese Patients Who Undergo Laparoscopic Colectomy for Benign or Malignant Disease of the Colon? Surgery, 40, 
675-682. https://doi.org/10.1016/j.surg.2006.07.013

[21] Bergamaschi, R., et al. (2008) Standardized Laparoscopic Intracorporeal Right Colectomy for Cancer: Short-Term Outcome in 111 Unselected Patients. Diseases of the Colon \& Rectum, 51, 1350-1355. https://doi.org/10.1007/s10350-008-9341-1

[22] Hanna, M.H., et al. (2016) Laparoscopic Right Hemicolectomy: Short and Long-Term Outcomes of Intracorporeal versus Extracorporeal Anastomosis. Surgical Endoscopy, 30, 3933-3942. https://doi.org/10.1007/s00464-015-4704-x

[23] Gil, I.C., et al. (2018) Intracorporeal versus Extracorporeal Anastomosis in Laparoscopic Right Colectomy: Short-Term Outcomes. Surgery, Gastroenterology and Oncology, 23, 71-78. https://doi.org/10.21614/sgo-23-1-71

[24] Fabozzi, M., et al. (2010) Comparison of Short- and Medium-Term Results between Laparoscopically Assisted and Totally Laparoscopic Right Hemicolectomy: A CaseControl Study. Surgical Endoscopy, 24, 2085-2091.

https://doi.org/10.1007/s00464-010-0902-8

[25] Carnuccio, P., Jimeno, J. and Pares, D. (2014) Laparoscopic Right Colectomy: A Systematic Review and Meta-Analysis of Observational Studies Comparing Two Types of Anastomosis. Techniques in Coloproctology, 18, 5-12. https://doi.org/10.1007/s10151-013-1029-4

[26] Winslow, E.R., et al. (2002) Wound Complications of Laparoscopic vs. Open Colectomy. Surgical Endoscopy, 16, 1420-1425.

https://doi.org/10.1007/s00464-002-8837-3

[27] Singh, R., et al. (2008) Does the Extraction-Site Location in Laparoscopic Colorectal Surgery Have an Impact on Incisional Hernia Rates? Surgical Endoscopy, 22, 25962600. https://doi.org/10.1007/s00464-008-9845-8

[28] Kisielinski, K., et al. (2004) The Pfannenstiel as So Called "Bikini Cut": Still Effective More than 100 Years after First Description. Hernia, 8, 177-181.

https://doi.org/10.1007/s10029-004-0210-0 